Check for updates

Cite this: RSC Adv., 2019, 9, 33282

Received 13th August 2019

Accepted 7th October 2019

DOI: $10.1039 / \mathrm{c} 9 \mathrm{ra06310b}$

rsc.li/rsc-advances

\section{Thermal stability, electrochemical and structural characterization of hydrothermally synthesised cobalt ferrite $\left(\mathrm{CoFe}_{2} \mathrm{O}_{4}\right)$}

\author{
Michele Bastianello, (D) abc Silvia Gross (D) ${ }^{c}$ and Matthias T. Elm (D) *abd
}

\begin{abstract}
Monophasic nano-crystalline $\mathrm{CoFe}_{2} \mathrm{O}_{4}$ (CFO) nanoparticles of high purity have been synthesised through a low temperature hydrothermal route, which does not involve hazardous chemicals, or conditions. The easy, green procedure involves a hydrothermal treatment at $135{ }^{\circ} \mathrm{C}$ of an aqueous suspension of the oxalate salts of the precursors. No further purification or annealing procedure was necessary to obtain the crystalline nano-structured oxide. The nanoparticles were characterized structurally and chemically by powder X-ray diffraction (PXRD), Inductively Coupled Plasma Spectrometry (ICP-MS) and Scanning Electron Microscopy (SEM), thus confirming the successful synthesis of the $\mathrm{CoFe}_{2} \mathrm{O}_{4}$ particles with the expected crystal phase and stoichiometry and an almost complete inverse spinel structure. From the nanoparticles pellets were pressed to investigate the electronic conduction properties using electrochemical impedance spectroscopy (EIS). At low temperatures, the conductivity measurements reveal a semiconducting behavior originating from hopping between Co sites and a total conductivity dominated by the grain boundary contribution. At higher temperatures $\left(T>400^{\circ} \mathrm{C}\right.$ ) a metallic-insulator transition occurs, which is attributed to additional hopping of electrons between the Fe sites.
\end{abstract}

\section{Introduction}

Due to their magnetic, electronic, and dielectric properties, transition metal spinel ferrites are of great importance for modern electronic industry and have been studied intensively in the last century. ${ }^{\mathbf{1 - 4}}$ The material properties can be tailored over a wide range by changing the oxide composition and its structural features. The high tunability of the properties arises from doping of the spinel structure with the general formula $\left[\mathrm{M}_{(1-\delta)}^{(\mathrm{II})} \mathrm{Fe}_{\delta}^{(\mathrm{III})}\right]_{\mathrm{Td}}\left[\mathrm{M}_{\delta}^{(\mathrm{II})} \mathrm{Fe}_{(2-\delta)}^{(\mathrm{III})}\right]_{\mathrm{Oh}} \mathrm{O}_{4}$, where the $\mathrm{M}^{(\mathrm{II})}$ and $\mathrm{Fe}^{(\mathrm{III})}$ cations are coordinated by oxide ions in 8 out of 64 tetrahedral $\left(T_{\mathrm{d}}\right)$ or 16 out of 32 octahedral $\left(O_{\mathrm{h}}\right)$ sites per unit cell. A normal spinel ferrite (e.g. bulk $\mathrm{Zn}^{(\mathrm{II})} \mathrm{Fe}_{2}^{(\mathrm{III})} \mathrm{O}_{4}$ ) has all the $\mathrm{M}^{(\mathrm{II})}$ in $T_{\mathrm{d}}$ coordination sites and all the $\mathrm{Fe}^{(\mathrm{III})}$ in $O_{\mathrm{h}}$ sites, while an inverse spinel (e.g. bulk $\mathrm{Ni}^{(\mathrm{II})} \mathrm{Fe}_{2}^{(\mathrm{III})} \mathrm{O}_{4}$ ) has all the $\mathrm{M}^{\text {(II) }}$ ions sitting in $O_{\mathrm{h}}$ sites and half $\mathrm{Fe}^{(\mathrm{III})}$ sitting in $O_{\mathrm{h}}$ and half in $T_{\mathrm{d}}$ sites. Bulk $\mathrm{CoFe}_{2} \mathrm{O}_{4}$ (CFO) in particular is a ferrimagnetic semiconducting material with an inverse spinel structure with $\mathrm{Co}^{(\mathrm{II})}$ sitting on the octahedral sites. ${ }^{\mathbf{5 , 6}}$ Amongst others CFO exhibits a high

${ }^{a}$ Center for Materials Research (LaMa), Justus-Liebig-Universität Gießen, Heinrich-Buff-Ring 16, 35392 Giessen, Germany

${ }^{b}$ Institute of Physical Chemistry, Justus-Liebig-Universität Gießen, Heinrich-Buff-Ring 17, 35392 Giessen, Germany.E-mail: matthias.elm@phys.chemie.uni-giessen.de ${ }^{c}$ Dipartimento di Scienze Chimiche, Università Degli Studi di Padova, 3100 Padova, Italy

${ }^{d}$ Institute of Experimental Physics I, Justus-Liebig-Universität Gießen, Heinrich-BuffRing 17, 35392 Giessen, Germany coercivity, Curie temperature, and chemical stability, ${ }^{7-11}$ making CFO an interesting material for many applications, such as recording devices, microwave absorption electro-magnetic wave shielding devices, sensors, spintronic applications, and as electrodes in $\mathrm{Li}$ ion batteries., ${ }^{\mathbf{1 1 2 - 1 7}}$ In the last years, ferrite nanoparticles especially have attracted much attention as their properties are not only affected by the chemical composition, but also by the size of the crystallites. ${ }^{6,18-23}$ For example, in CFO nanoparticles an increase of the coercivity was reported for decreasing particle size. ${ }^{6,22}$ Not only the magnetic properties, but also the electrical properties vary with size, shape and the preparation condition of the particles. ${ }^{\mathbf{6 , 8 , 2 4 , 2 5}}$ The reason is that nano-structured oxide ferrites rarely exhibit a pure inverse or direct spinel structure. ${ }^{26,27}$ Most of the prepared ferrites have a partial inversion degree $\delta$ in its crystal structure, which depends on the preparation procedure and on the further treatment of the oxide, i.e. if $\delta$ is equal to 0 the spinel is direct and the $\mathrm{M}^{(\mathrm{II})}$ are sitting only on $T_{\mathrm{d}}$ sites, while the $\mathrm{Fe}^{(\mathrm{III})}$ occupy only the $O_{\mathrm{h}}$ sites. Some of us have recently shown that the synthesis route can remarkably affect the inversion degree. ${ }^{28}$ To synthesise ferrite nanoparticles several methods have been developed during the last years. Solid-states methods, such as mechanical milling ${ }^{29,30}$ are advantageous in scalability and in absence of purification steps, especially for industrial scale up. Nevertheless, the limited contact area between the reactant causes undesired sub-products and slow reaction rates are needed to be compensated with increased temperature of about $800{ }^{\circ} \mathrm{C}^{31-34}$ Amongst wet-chemical methods, including sol-gel, 
co-precipitation or thermal composition, hydrothermal synthesis is an important low temperature alternative as structurally uniform particles can be synthesised at temperatures below $150{ }^{\circ} \mathrm{C} \cdot{ }^{8,31,34,35}$ However, due to the strong dependence of the electrical and magnetic properties of ferrites on their structural properties and degree of inversion a detailed characterization of the nanoparticles synthesized by hydrothermal methods is essential to optimize the materials' properties for future applications.

Here, we present the preparation and characterization of nanostructured monophasic CFO using a wet chemistry route based on water as solvent and mild reaction conditions, and affording the formation of the ferrites in extremely short treatment time (i.e. 1-3 hours). ${ }^{36,37}$ The synthetic route employed is designed to take into account most of the green chemistry principles ${ }^{38}$ such as atom economy, a low temperature or energy efficient process, and the use of non-hazardous solvents and chemicals to have an environmentally friendly alternative to the solid-state synthesis for the production of mixed oxides. Monophasic nanostructured CFO with a crystalline domain size of $15-20 \mathrm{~nm}$ were prepared in high yield and high atomic efficiency using water as solvent and a mild thermal treatment $\left(135^{\circ} \mathrm{C}\right)$. The $\mathrm{CFO}$ nanoparticles were treated at higher temperatures to perform dielectric characterization. To investigate the influence of the heat treatment and the resulting change in microstructure on the electrical transport properties, two different temperatures of $400{ }^{\circ} \mathrm{C}$ and $950{ }^{\circ} \mathrm{C}$ were chosen. Structural investigations reveal that nanoparticles with a nearly complete inverse spinel structure are obtained. The analysis of the electrical transport properties using electrochemical impedance spectroscopy shows a semiconducting behavior due to hole hopping between Co sites at temperatures below $400{ }^{\circ} \mathrm{C}$, while a semiconductor to metal transition occurs at higher temperature due to additional electron hopping between Fe sites of the lattice.

\section{Results and discussion}

\section{High-resolution scanning electron microscopy}

The microstructure of the pellets prepared was analyzed using High-resolution scanning electron microscopy (HR-SEM) after the annealing process. As shown in Fig. 1, both pellets annealed
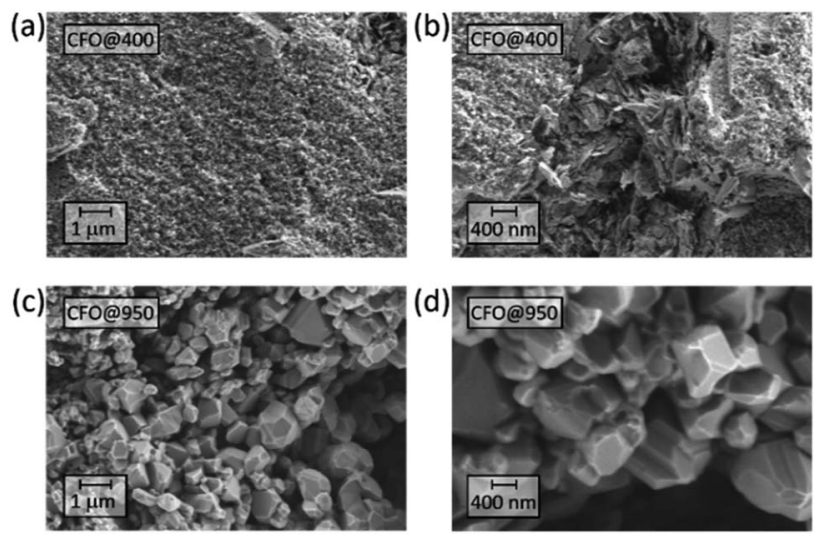

Fig. 1 HR-SEM of CFO@400 ( $a$ and b) and CFO@950 (c and d). at $400{ }^{\circ} \mathrm{C}$ (CFO@400) and $950{ }^{\circ} \mathrm{C}$ (CFO@950) reveal a complex microstructure, whereas the pellet annealed at $950{ }^{\circ} \mathrm{C}$ is denser than the one annealed at $400{ }^{\circ} \mathrm{C}$. Furthermore, beside single nanoparticles with diameters of about $20 \mathrm{~nm}$ also large grains with diameters up to $1 \mu \mathrm{m}$ can be observed for the sample annealed at $400{ }^{\circ} \mathrm{C}$. With increasing annealing temperature, the average diameter of the single grains increases resulting in different crystalline domains of several $\mu \mathrm{m}$ for the pellet annealed at $950{ }^{\circ} \mathrm{C}$.

\section{XRD analysis}

In order to determine the crystal structure of the obtained material, X-ray powder diffraction measurements were performed, which are shown in Fig. 2. The labeled reflections denote the reflections of a cubic Bravais lattice with a $F d \overline{3} m$ space group, the $\mathrm{CoFe}_{2} \mathrm{O}_{4}$ reference pattern ICSD 98553 (ref. 39) is shown for comparison. All patterns show the characteristic reflections of cubic CFO. For the as-prepared sample (CFO@RT) as well as the pellet annealed at $400{ }^{\circ} \mathrm{C}$ (CFO@400) the diffraction pattern shows an additional, weak reflections at $49.7^{\circ}$, which probably arises due to a negligible, secondary phase as by-product. This additional reflection is not present in the pattern of the pellet annealed at $950{ }^{\circ} \mathrm{C}$ (CFO@950) confirming in this case a phase pure material. Compared to the CFO@RT pattern, the reflections of the CFO@400 sample did not change, while the CFO@950 pellet shows extremely sharp reflections due to the size increase of the crystalline domains during the annealing process. Using the Scherrer equation ${ }^{40}$ on different reflections $(h k l)$,

$$
d_{(h k l)}=\frac{K \lambda}{\beta_{(h k l)} \cos \theta_{(h k l)}}
$$

being $d_{(h k l)}$ the supposedly spherical crystallite dimension, $\beta_{(h k l)}$ the full width at half maximum, $\theta_{(h k l)}$ the half of the reflection diffraction angle. The particles sizes for the CFO@RT, CFO@400 and CFO@950 sample were estimated to be $16.7 \pm$ $4 \mathrm{~nm}, 17.6 \pm 4 \mathrm{~nm}$ and $46.4 \pm 8 \mathrm{~nm}$, respectively. Thus, the

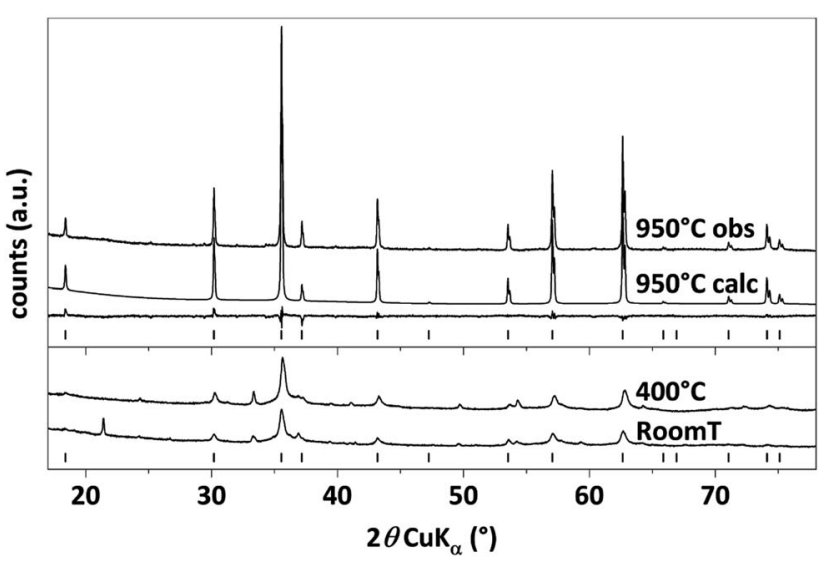

Fig. 2 PXRD of CFO@RT, CFO@400 and CFO@950. Calculated pattern from the CFO@950 Rietveld refinement and its difference from the observed data. The reference pattern ICSD 98553 is also shown for comparison. 
growth of the crystallites occurs between 400 and $950{ }^{\circ} \mathrm{C}$. It is worth noting that the Scherrer equation is less reliable for crystallites bigger than $100 \mathrm{~nm}$ because at these dimensions the widening of the reflections is caused by instrumental limitations.

To refine the atomic positions in the lattice and, therefore, to investigate the occurrence of a correlation between structural features and electrical behaviour, Rietveld refinement was performed on the CFO@950 pattern. A Chebyshev function ${ }^{41}$ with ten coefficients was used to fit the pattern baseline. Only the $T_{\mathrm{d}}$ and $O_{\mathrm{h}}$ fractional position and Uiso parameter for both ions $\mathrm{Co}^{2+}$ and $\mathrm{Fe}^{3+}$ were refined applying the identity restriction to the same site type and the sum to complementary multiplicity restriction to the sites in the unit cell, while the oxygen position and fraction (Frac) were not refined.

The resulting fit scores a goodness-of-fit (GOF) equal to 1.51 and can be considered as reliable. ${ }^{42}$ As shown in Table 1 , the occupancy value for the metallic ions indicates a partial inversion $\delta$ of 0.91 indicating an almost completely inverse spinel. On average, $46 \%$ of the $O_{\mathrm{h}}$ sites of the crystalline domain are occupied by $\mathrm{Co}^{2+}$ and $54 \%$ are occupied by $\mathrm{Fe}^{3+}$, i.e. there is a statistical alternation of $\mathrm{Co}^{2+}$ and $\mathrm{Fe}^{3+}$ in adjacent $O_{\mathrm{h}}$ positions.

\section{ICP-MS}

To validate the PXRD analyses the actual chemical composition of the mixed oxide was determined using inductively coupled plasma mass spectrometry (ICP-MS).

As shown in Table 2, the experimentally determined stoichiometry of the oxide coincides with the theoretical one within the experimental error range, thus confirming the reliability of the synthesis route.

\section{Impedance measurements}

To investigate the electrical properties of the CFO pellets, temperature-dependent impedance measurements were performed. Fig. 3(a) shows the impedance spectra in the Nyquist plots of the CFO@950 pellet, which exhibits two distinct semicircles in the temperature range between $-45{ }^{\circ} \mathrm{C}$ and $50{ }^{\circ} \mathrm{C}$. To analyze the impedance data an equivalent circuit with two $R Q$ elements connected in series was used. Each $R Q$ element consists of a resistance $R$ and a constant phase elements (CPE) $Q$ representing one of the non-ideal semicircles. According to the brick layer model of microcrystalline ceramic materials, ${ }^{\mathbf{4 3 - 4 7}}$ the semicircle in the high frequency range is interpreted as the

Table 1 Metal ions occupancies of CFO@950 obtained from Rietveld refinement of the PXRD diffraction pattern

\begin{tabular}{llllr}
\hline Ion & Site & Frac & Uiso & GoF \\
\hline $\mathrm{Co}(\mathrm{II}))_{T_{\mathrm{d}}}$ & $T_{\mathrm{d}}$ & 0.09 & $2 \times 10^{-4}$ & 1.51 \\
$\mathrm{Fe}(\mathrm{III})_{T_{\mathrm{d}}}$ & $T_{\mathrm{d}}$ & 0.91 & $2 \times 10^{-4}$ & \\
$\mathrm{Co}(\mathrm{II})_{O_{\mathrm{h}}}$ & $O_{\mathrm{h}}$ & 0.46 & $2.1 \times 10^{-3}$ & \\
$\mathrm{Fe}(\mathrm{III}) O_{O_{\mathrm{h}}}$ & $O_{\mathrm{h}}$ & 0.54 & $2.1 \times 10^{-3}$ & \\
$\mathrm{O}$ & 32 & 1.00 & $1.2 \times 10^{-2}$ &
\end{tabular}

Table 2 Theoretical and experimental stoichiometry of the hydrothermally synthetised CFO

\begin{tabular}{ll}
\hline Expected stoichiometry & $\begin{array}{l}\text { Experimental } \\
\text { stoichiometry }\end{array}$ \\
\hline $\mathrm{CoFe}_{2} \mathrm{O}_{4}$ & $\mathrm{Co}_{1.001} \mathrm{Fe}_{2.008} \mathrm{O}_{(4+\delta)}$ \\
\hline
\end{tabular}

transport process inside the crystallites, i.e. the bulk properties, while the semicircle at lower frequencies represents the transport process across the grain boundaries. At higher temperature

(a)
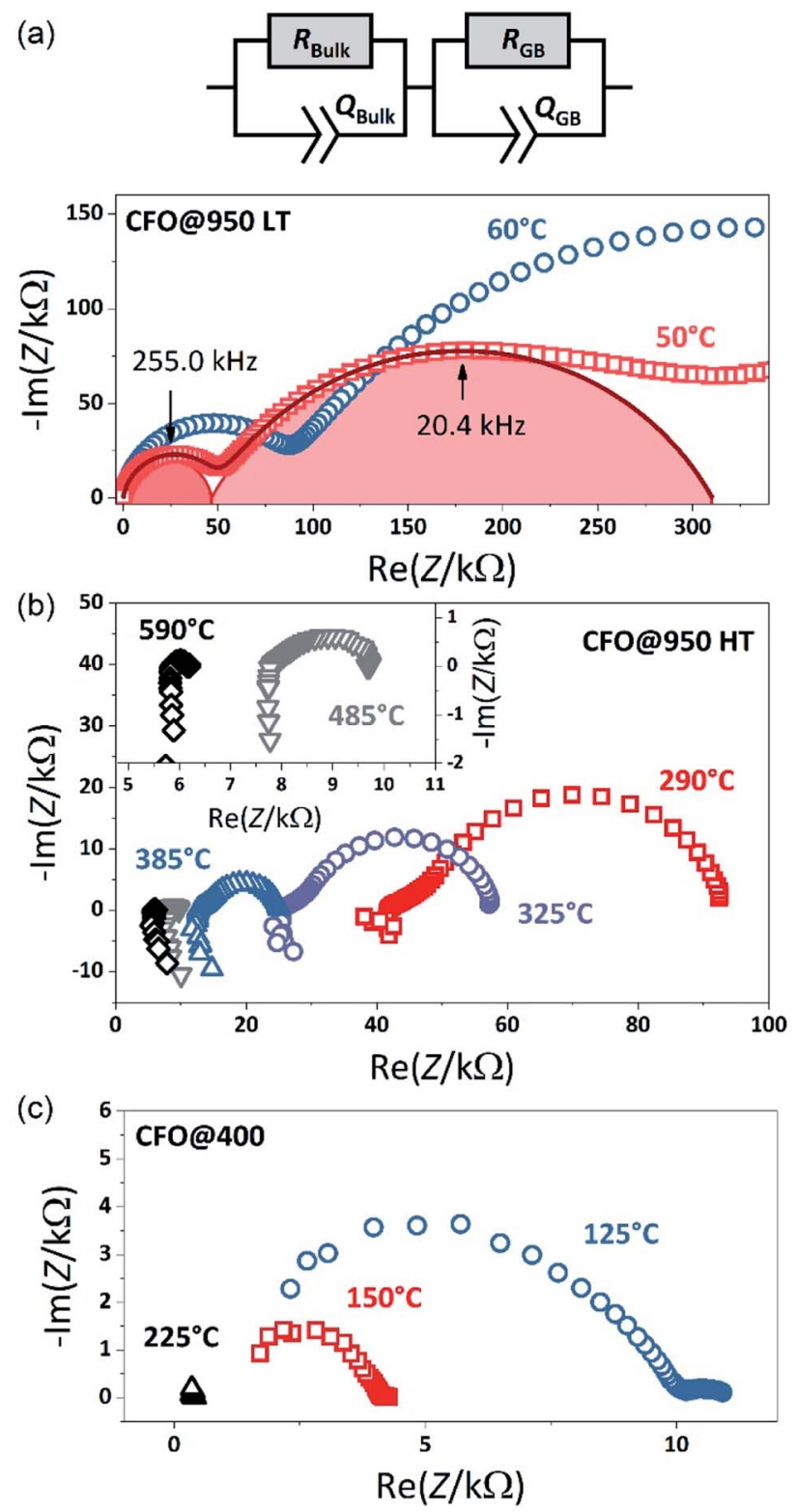

Fig. 3 (a) Nyquist plot of the impedance spectrum of CFO@950 measured in the temperature range between -45 and $70{ }^{\circ} \mathrm{C}$ (just 60 and $50^{\circ} \mathrm{C}$ shown) and the equivalent circuit used to describe the data. Nyquist plot of the impedance spectrum of (b) CFO@950 measured between 290 and $590^{\circ} \mathrm{C}$, and (c) of CFO@400 measured between 125 and $225^{\circ} \mathrm{C}$. 
$\left(T>50{ }^{\circ} \mathrm{C}\right)$ the onset of an additional transport process, i.e. a third semi-circle, is observed which is caused by the electric charge transfer at the Pt electrode as often observed for oxide materials. ${ }^{48,49}$ However, this third semi-circle is not considered in the fitting to ensure continuity between the different temperature regimes by using the same equivalent circle. The corresponding fits to the data are shown in Fig. 3 as solid lines. From the constant phase elements $Q_{\mathrm{i}}$ the capacitance of the corresponding transport process can be calculated: ${ }^{\mathbf{4 9 - 5 1}}$

$$
C_{\mathrm{i}}=R_{\mathrm{i}}^{(1-n)} Q_{\mathrm{i}}^{1 / n}
$$

For the semicircle in the high frequency range a capacitance $C_{1}$ of about $1.2-1.4 \times 10^{-11} \mathrm{~F}$ is obtained, which is a typical value for the bulk material. ${ }^{\mathbf{4 3 4 5}}$ The second semicircle shows a capacitance of about $5 \times 10^{-10} \mathrm{~F}$. The second semicircle therefore can be attributed to the response of the grain boundaries, which show values between $C_{2}=10^{-10}$ to $10^{-8} \mathrm{~F}$ depending on the size of the grains in the particle, ${ }^{46,52,53}$ i.e. with decreasing grain size the capacity shifts to lower values resulting in an overlap of the bulk and grain boundary semicircle in nanocrystalline samples. ${ }^{\mathbf{4 6 , 4 9 , 5 2}}$ Not only the grain size, but also the temperature can result in a merging of the two semicircles due to the temperature dependence of the resistance as shown in Fig. 3(b). With increasing temperature, the bulk and the grain boundary resistances decrease resulting in a dominant grain boundary contribution and, finally, only an ohmic behavior is observed for temperatures above $600{ }^{\circ} \mathrm{C}$ accompanied with inductive contributions from the measurement setup. Thus, in the temperature range between $140{ }^{\circ} \mathrm{C}$ and $700{ }^{\circ} \mathrm{C}$ only the total resistance was extracted from the impedance spectra.

Fig. 3(c) finally shows the impedance spectra obtained from the CFO@400 pellet measured $50{ }^{\circ} \mathrm{C}$ and $130{ }^{\circ} \mathrm{C}$. Compared to the CFO@950 pellet, only the tail of one semicircle is observed, representing the grain and the grain boundary contribution, accompanied with a small semicircle at low frequencies again arising from the electrode. Thus, the total resistance of the sample is obtained at the intercept point of the first semicircle on the $x$-axis. The merging of the semicircles at high frequencies representing the grain and the grain boundary contribution imply that the crystallites are in the nanosize range ${ }^{\mathbf{4 6 , 4 9 , 5 4}}$ in agreement with the SEM and XRD observation.

In magnetite the electronic conductivity above the Verwey transition arises from polaron hopping of the electrons between on the octahedral coordinated $\mathrm{Fe}_{\mathrm{B}}{ }^{2+}$ and $\mathrm{Fe}_{\mathrm{B}}{ }^{3+}$ sites centres throughout the oxide. ${ }^{14,55,56}$ Although $\mathrm{CoFe}_{2} \mathrm{O}_{4}$ has a similar crystal structure as magnetite, replacing $\mathrm{Fe}_{\mathrm{B}}{ }^{2+}$ with $\mathrm{Co}_{\mathrm{B}}{ }^{2+}$ reduces the electrical conductivity as cobalt prefers a $\mathrm{Co}^{2+}$ valence state and has no weakly bound electrons, which hinders electron hopping between the octahedral sites. ${ }^{14}$ Instead, hole hopping between $\mathrm{Co}_{\mathrm{B}}{ }^{3+}$ and $\mathrm{Co}_{\mathrm{B}}{ }^{2+}$ can occur. In general, the total conductivity in CFO is therefore given by: ${ }^{57}$

$$
\sigma=\sigma_{\mathrm{e}}+\sigma_{\mathrm{h}}=n_{\mathrm{e}} e \mu_{\mathrm{e}}+n_{\mathrm{h}} e \mu_{\mathrm{h}}
$$

with $\sigma_{\mathrm{e}}$ and $\sigma_{\mathrm{h}}$ being the electron and hole conductivity, $e$ is the elementary charge, and $n_{\mathrm{e}, \mathrm{h}}$ and $\mu_{\mathrm{e}, \mathrm{h}}$ are the corresponding carrier concentrations and mobilities, respectively. Which of both conductivities dominates the overall transport behavior of cobalt-ferrite strongly depends on the composition as well as on the stoichiometry. While $\mathrm{Co}_{1-x} \mathrm{Fe}_{2+x} \mathrm{O}_{4}$ exhibits n-type conductivity, where electron hopping between $\mathrm{Fe}_{\mathrm{B}}{ }^{2+}$ and $\mathrm{Fe}_{\mathrm{B}}{ }^{3+}$ sites dominates, $\mathrm{CFO}$ with iron excess $\left(\mathrm{Co}_{1+x} \mathrm{Fe}_{2-x} \mathrm{O}_{4}\right)$ shows p-type conductivity due to hole hopping between $\mathrm{Co}_{\mathrm{B}}{ }^{2+}$ and $\mathrm{Co}_{\mathrm{B}}{ }^{3+} \cdot{ }^{57,58}$

To analyse the conduction process in more detail, the activation energy was determined from the temperature dependence of the conductivity. Fig. 4 shows the conductivity of both pellets in an Arrhenius-type representation. Both pellets show in the temperature range below $400{ }^{\circ} \mathrm{C}$ a semiconducting behavior, i.e. an increase in conductivity with increasing temperature, which is typical for hopping transport in ferrites. ${ }^{17,58-61}$ The temperature-dependence of the conductivity is then given by:

$$
\sigma(T)=\frac{\sigma_{0}}{T} \exp \left(-\frac{E_{\mathrm{A}}}{k_{\mathrm{B}} T}\right)
$$

where $E_{\mathrm{A}}$ denotes the activation energy of the hopping process. Fitting the experimental data of the CFO@950 sample to the expression $\ln (\sigma T)=\ln \left(\sigma_{0}\right)-E_{\mathrm{A}} / k_{\mathrm{B}} T$ gave an activation energy of $0.50 \pm 0.015 \mathrm{eV}$ and $0.64 \pm 0.02 \mathrm{eV}$ for the bulk and the grain boundary contribution, respectively. The bulk conductivity is two magnitudes larger than the grain boundary conductivity, i.e. the grain boundaries act as barriers for the electronic transport. The total conductivity and the corresponding activation energy $\left(E_{\mathrm{A}}=0.62 \pm 0.02 \mathrm{eV}\right)$ of the pellet are therefore dominated by the grain boundaries. As discussed above, the bulk and the grain boundary contribution could only be separated below $100{ }^{\circ} \mathrm{C}$. Thus, at higher temperature only the activation energy of the total conductivity was determined with $E_{\mathrm{A}}=$ $0.63 \pm 0.02 \mathrm{eV}$, which is in excellent agreement with the low temperature value. The activation energy obtained is also comparable to values reported in literature for nanocrystalline

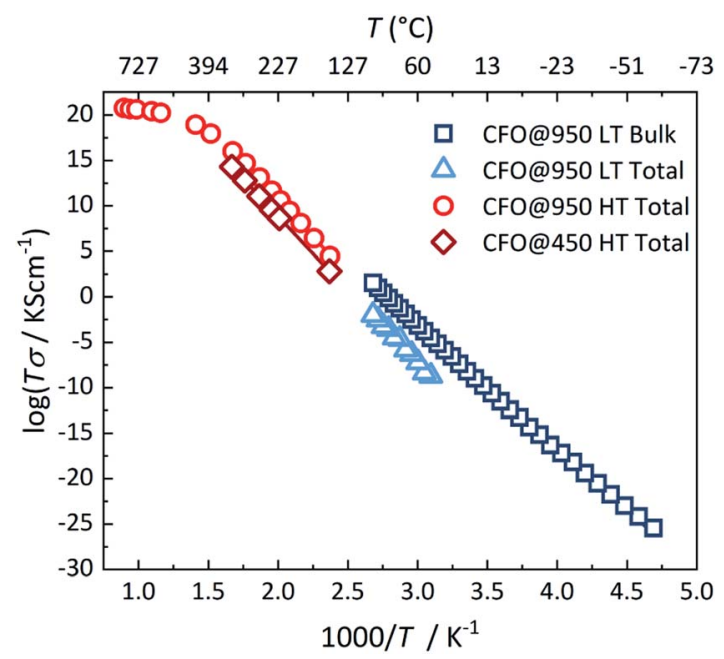

Fig. 4 Arrhenius plot of the conductivities of CFO@400 and CFO@950 
$\mathrm{CoFe}_{2} \mathrm{O}_{4}$, which are in the range between 0.55 to $0.65 \mathrm{eV}^{.57,58,62,63}$ Furthermore, it reveals that hole hopping between the Co sites is the dominant transport mechanism at low temperatures as the activation energy for electron hopping between Fe sites is in the range of $0.2 \mathrm{eV} \cdot{ }^{57-59}$ For the CFO@400 pellet the same activation energy was determined. However, the total conductivity is about two orders of magnitude smaller than for the CFO@950 sample which is due to the smaller grains of the sample and the resulting larger grain boundary contribution. Above $300{ }^{\circ} \mathrm{C}$, the slope of the total conductivity of the CFO@950 sample changes and a much smaller activation energy of about $0.17 \pm 0.014 \mathrm{eV}$ is observed. This behavior is attributed to a change in the transport mechanism. As discussed above holes as well as electrons contribute to the transport in cobalt-ferrites, whereas each can dominate the overall behavior depending on the composition and the stoichiometry of the ferrite. With increasing temperature, the cobalt ferrite is reduced, i.e. oxygen is released from the lattice, resulting in an increased density of electrons due to charge compensation. Thus, at high temperatures also electron hopping between the $\mathrm{Fe}_{\mathrm{B}}{ }^{2+}$ and $\mathrm{Fe}_{\mathrm{B}}{ }^{3+}$ site centres takes place. As the electron mobility is larger than the hole mobility, the overall transport behavior at high temperatures is dominated by the electronic conductivity resulting in the observed decrease of the activation energy in agreement with previous reports. ${ }^{62,63}$

\section{Experimental}

\section{Chemicals}

Tetraethylammonium hydroxide $(20 \% \mathrm{w} / \mathrm{w}$ in water) (TENOH) and sodium hydroxide were purchased from Sigma Aldrich (Milan, Italy). Iron(III) nitrate nonahydrate was purchased from Merck (Darmstadt, Germany). Oxalic acid dihydrate (99.8\%) and cobalt(II) chloride hexahydrate were purchased from Carlo Erba (Rodano, Milan, Italy).

\section{Sample preparation}

According to the previously reported procedure ${ }^{37}$ stoichiometric amount of the precursor metal salts and oxalic acid were dissolved in $10 \mathrm{~mL}$ deionised water in a $23 \mathrm{~mL}$ Teflon liner. The concentration of iron(III) and cobalt(II) were kept at $\sim 0.1 \mathrm{~mol} \mathrm{~L}^{-1}$ and $\sim 0.05 \mathrm{~mol} \mathrm{~L}^{-1}$, respectively. $0.2 \mathrm{~mL}(0.14$ $\mathrm{mmol}$ ) of TENOH, acting as a peptising agent, were added to the solution. The precipitation of the precursor oxalate and hydroxides was induced by adding $1.5 \mathrm{~mL}$ of a $10 \mathrm{~mol} \mathrm{~L}^{-1} \mathrm{NaOH}$ aqueous solution achieving a $\mathrm{pH}$ of $12-13$. The liner was then sealed and placed in a $23 \mathrm{~mL}$ Parr stainless steel acid digestion bomb, heated at $135{ }^{\circ} \mathrm{C}$ for 72 hours and left to cool down to room temperature. The obtained solid powders were isolated and purified by washing and centrifugation in three cycles, the first two at 5000 rotations per minute (RPM) for 5 minutes and the last one at $12500 \mathrm{rpm}$ for 12.5 minutes. Finally, the nanoparticles were dried at $80{ }^{\circ} \mathrm{C}$. To perform electrochemical impedance spectroscopy measurements approximately $0.5 \mathrm{~g}$ of the sample powder was pressed to pellets with a diameter of $13 \mathrm{~mm}$ using a uniaxial press at $30 \mathrm{kN}$ force for five minutes and subsequently an isostatic press at 550 bar for one hour. Two as prepared pellets were sintered in air for 12 hours at $400^{\circ}$ and $955^{\circ} \mathrm{C}$, respectively, in a Heraeus D-6450 oven.

\section{PXRD analyses}

For the PXRD analysis a Panalytical Empyrean diffractometer was used with an angular accuracy of $0.001^{\circ}$ and an angular resolution better than 0.01 employing the $\mathrm{CuK}_{\alpha}$ radiation. The $\mathrm{X}$-ray patterns were collected with a $\lambda$ of $1.5406 \AA$, a scan step of $0.013^{\circ}(2 \theta)$ and 200 seconds acquisition per step time between 10 and $80^{\circ}$. The XRD profile was fitted using GSAS software.

\section{ICP-MS analyses}

For the ICP-MS analyses, all reagents were of analytical grade and used as purchased: nitric acid $(69 \% \mathrm{w} / \mathrm{w}$ Aristar for trace analysis, product key $450042 \mathrm{~N}$, batch K43187145 206). All solutions were prepared in milliQ ultrapure water obtained using a Millipore Plus System (Milan, Italy, resistivity 18.2 MOhm $\mathrm{cm}^{-1}$ ). The material weighted in using a five decimal digits analytical scale $(\mathrm{g})$, digested in nitric acid (69\% w/w) using the microwave digester CEM EXPLORER SPD 24/48 PLUS. Agilent's 7700 Series ICP-MS was tuned using a calibration standard (VI), 30 Analytes ULTRA SCIENTIFIC multi-standard ICPMS IMS-120 tuning solution prepared in 3\% $\mathrm{w} / \mathrm{w}$ nitric acid was used as an internal standard and added to the sample solution via a T-junction. The tuning range was between $25 \mathrm{ppb}$ and $1.5 \mathrm{ppm}$ for Co and between $250 \mathrm{ppb}$ and $14 \mathrm{ppm}$ for Fe.

\section{EIS analyses}

Electrical contact on the pellets were prepared by painting Ferro E1192 platinum paste on the two opposite circular faces of the pellets. The measurements were performed using a Parstat 2273 potentiostat coupled with a Nabertherm p320 furnace regulated with an Eurotherm 2416 thermostat for the measurements in the high temperature range $\left(140\right.$ to $\left.740{ }^{\circ} \mathrm{C}\right)$. In the low temperature range $\left(-50\right.$ to $\left.70{ }^{\circ} \mathrm{C}\right)$, a Novocool Cryosystem setup was used. The spectra were acquired between $100 \mathrm{mHz}$ and 1 $\mathrm{GHz}$ with the Parstat 2273 and between $100 \mathrm{mHz}$ and $2 \mathrm{GHz}$ with the Novocool Cryosystem setup. The analysis of the data and the fitting procedure were performed using the Relaxys software.

\section{SEM imaging}

The SEM images were acquired with a Zeiss Merlin microscope at a voltage between 13 and $10 \mathrm{kV}$ and a current between 90 and 187 pA.

\section{Conclusions}

Crystalline $\mathrm{CoFe}_{2} \mathrm{O}_{4}$ nanoparticles were successfully synthesized using a low temperature green hydrothermal synthesis route. Structural analyses confirm a nearly complete inverse spinel structure and stoichiometry of the nanoparticles with sizes ranging between 15 and $20 \mathrm{~nm}$. Impedance measurements on pellets as a function of temperature reveal that the total conductivity at temperatures below $400{ }^{\circ} \mathrm{C}$ is dominated by the 
grain boundary contribution with an activation energy of about $0.63 \mathrm{eV}$, which arises from hole hopping between Co sites. At higher temperatures the activation energy strongly decreases to $0.17 \mathrm{eV}$ due to an additional transport process of electron hopping between the Fe sites of the lattice.

\section{Conflicts of interest}

There are no conflicts to declare.

\section{Acknowledgements}

The authors gratefully acknowledge the Erasmus + program for the funding. M. B. and M. T. E. thank the German Federal Ministry of Education and Research (BMBF) for funding of the NanoMatFutur project NiKo (03XP0093). Prof. Denis Badocco and Prof. Paolo Pastore (Dipartimento di Scienze Chimiche, University of Padova) are gratefully acknowledged for ICP-MS measurements. S. G. acknowledges the DFG and the JustusLiebig Universität Gießen for the provision of a Mercator Fellowship (2016-2020) within the GrK (Research training group) 2204 "Substitute Materials for sustainable Energy Technologies”.

\section{Notes and references}

1 M. Sugimoto, The Past, Present, and Future of Ferrites, J. Am. Ceram. Soc., 1999, 82, 269-280.

2 Y. Suzuki, Epitaxial Spinel Ferrite Thin Films, Annu. Rev. Mater. Res., 2001, 31, 265-289.

3 J. D. Adam, L. E. Davis, G. F. Dionne, E. F. Schloemann and S. N. Stitzer, Ferrite devices and materials, IEEE Trans. Microwave Theory Tech., 2002, 50, 721-737.

4 V. G. Harris, A. Geiler, Y. Chen, S. D. Yoon, M. Wu, A. Yang, Z. Chen, P. He, P. V Parimi, X. Zuo, C. E. Patton, M. Abe, O. Acher and C. Vittoria, Recent advances in processing and applications of microwave ferrites, J. Magn. Magn. Mater., 2009, 321, 2035-2047.

5 L. Horng, G. Chern, M. C. Chen, P. C. Kang and D. S. Lee, Magnetic anisotropic properties in $\mathrm{Fe}_{3} \mathrm{O}_{4}$ and $\mathrm{CoFe}_{2} \mathrm{O}_{4}$ ferrite epitaxy thin films, J. Magn. Magn. Mater., 2004, 270, 389-396.

6 K. Vasundhara, S. N. Achary, S. K. Deshpande, P. D. Babu, S. S. Meena and A. K. Tyagi, Size dependent magnetic and dielectric properties of nano $\mathrm{CoFe}_{2} \mathrm{O}_{4}$ prepared by a salt assisted gel-combustion method, J. Appl. Phys., 2013, 113, 194101.

7 W. Chen and W. Zhu, Preparation of Cobalt Ferrite Thick Films and Their Magnetic and Electrical Properties, J. Am. Ceram. Soc., 2011, 94, 1096-1100.

8 F. Zan, Y. Ma, Q. Ma, Y. Xu, Z. Dai, G. Zheng, M. Wu and G. Li, Magnetic and Impedance Properties of Nanocomposite $\mathrm{CoFe}_{2} \mathrm{O}_{4} / \mathrm{Co}_{0.7} \mathrm{Fe}_{0.3}$ and Single-Phase $\mathrm{CoFe}_{2} \mathrm{O}_{4}$ Prepared Via a One-Step Hydrothermal Synthesis, J. Am. Ceram. Soc., 2013, 96, 3100-3107.
9 T. M. Whitney, P. C. Searson, J. S. Jiang and C. L. Chien, Fabrication and Magnetic Properties of Arrays of Metallic Nanowires, Science, 1993, 261, 1316-1319.

10 S. R. Naik and A. V Salker, Change in the magnetostructural properties of rare earth doped cobalt ferrites relative to the magnetic anisotropy, J. Mater. Chem., 2012, 22, 2740-2750.

11 S. Gyergyek, D. Makovec, A. Kodre, I. Arčon, M. Jagodič and M. Drofenik, Influence of synthesis method on structural and magnetic properties of cobalt ferrite nanoparticles, $J$. Nanopart. Res., 2010, 12, 1263-1273.

12 D. Goll and H. Kronmüller, High-performance permanent magnets, Naturwissenschaften, 2000, 87, 423-438.

13 C. Xiangfeng, J. Dongli, G. Yu and Z. Chenmou, Ethanol gas sensor based on CoFe2O4 nano-crystallines prepared by hydrothermal method, Sens. Actuators, B, 2006, 120, 177181.

14 J. A. Moyer, C. A. F. Vaz, E. Negusse, D. A. Arena and V. E. Henrich, Controlling the electronic structure of $\mathrm{Co}_{1-}$ ${ }_{x} \mathrm{Zn}_{x} \mathrm{Fe}_{2} \mathrm{O}_{4}$ thin films through iron doping, Phys. Rev. B: Condens. Matter Mater. Phys., 2011, 83, 35121.

15 Z. H. Li, T. P. Zhao, X. Y. Zhan, D. S. Gao, Q. Z. Xiao and G. T. Lei, High capacity three-dimensional ordered macroporous $\mathrm{CoFe}_{2} \mathrm{O}_{4}$ as anode material for lithium ion batteries, Electrochim. Acta, 2010, 55, 4594-4598.

16 P. Lavela and J. L. Tirado, $\mathrm{CoFe}_{2} \mathrm{O}_{4}$ and $\mathrm{NiFe}_{2} \mathrm{O}_{4}$ synthesized by sol-gel procedures for their use as anode materials for $\mathrm{Li}$ ion batteries, J. Power Sources, 2007, 172, 379-387.

17 R. A. Henning, P. Uredat, C. Simon, A. Bloesser, P. Cop, M. T. Elm and R. Marschall, Characterization of $\mathrm{MFe}_{2} \mathrm{O}_{4}$ $(\mathrm{M}=\mathrm{Mg}, \mathrm{Zn})$ Thin Films Prepared by Pulsed Laser Deposition for Photoelectrochemical Applications, J. Phys. Chem. C, 2019, 123, 18240-18247.

18 L. Zhao, H. Zhang, Y. Xing, S. Song, S. Yu, W. Shi, X. Guo, J. Yang, Y. Lei and F. Cao, Studies on the magnetism of cobalt ferrite nanocrystals synthesized by hydrothermal method, J. Solid State Chem., 2008, 181, 245-252.

19 J. M. Soares, F. A. O. Cabral, J. H. de Araújo and F. L. A. Machado, Exchange-spring behavior in nanopowders of $\mathrm{CoFe}_{2} \mathrm{O}_{4}-\mathrm{CoFe}_{2}$, Appl. Phys. Lett., 2011, 98, 72502.

20 K. K. Kefeni, T. A. M. Msagati and B. B. Mamba, Ferrite nanoparticles: synthesis, characterisation and applications in electronic device, Mater. Sci. Eng., B, 2017, 215, 37-55.

21 R. H. Kodama, A. E. Berkowitz, E. J. McNiff and S. Foner, Surface Spin Disorder in $\mathrm{CoFe}_{2} \mathrm{O}_{4}$ Nanoparticles, Phys. Rev. Lett., 1996, 77, 394-397.

22 Q. Song and Z. J. Zhang, Shape control and associated magnetic properties of spinel cobalt ferrite nanocrystals, $J$. Am. Chem. Soc., 2004, 126, 6164-6168.

23 T.-J. Park, G. C. Papaefthymiou, A. J. Viescas, A. R. Moodenbaugh and S. S. Wong, Size-dependent magnetic properties of single-crystalline multiferroic $\mathrm{BiFeO}_{3}$ nanoparticles, Nano Lett., 2007, 7, 766-772.

24 N. Ponpandian, P. Balaya and A. Narayanasamy, Electrical conductivity and dielectric behaviour of nanocrystalline $\mathrm{NiFe}_{2} \mathrm{O}_{4}$ spinel, J. Phys.: Condens. Matter, 2002, 14, 32213237. 
25 T. J. Daou, G. Pourroy, S. Bégin-Colin, J. M. Grenèche, C. Ulhaq-Bouillet, P. Legaré, P. Bernhardt, C. Leuvrey and G. Rogez, Hydrothermal Synthesis of Monodisperse Magnetite Nanoparticles, Chem. Mater., 2006, 18, 4399-4404.

26 C. N. Chinnasamy, A. Narayanasamy, N. Ponpandian, K. Chattopadhyay, K. Shinoda, B. Jeyadevan, K. Tohji, K. Nakatsuka, T. Furubayashi and I. Nakatani, Mixed spinel structure in nanocrystalline $\mathrm{NiFe}_{2} \mathrm{O}_{4}$, Phys. Rev. B: Condens. Matter Mater. Phys., 2001, 63, 184108.

27 D. Carta, M. F. Casula, A. Falqui, D. Loche, G. Mountjoy, C. Sangregorio and A. Corrias, A Structural and Magnetic Investigation of the Inversion Degree in Ferrite Nanocrystals $\mathrm{MFe}_{2} \mathrm{O}_{4}(\mathrm{M}=\mathrm{Mn}, \mathrm{Co}, \mathrm{Ni}), J$. Phys. Chem. C, 2009, 113, 8606-8615.

28 P. Dolcet, K. Kirchberg, A. Antonello, C. Suchomski, R. Marschall, S. Diodati, R. Muñoz-Espí, K. Landfester and S. Gross, Exploring wet chemistry approaches to $\mathrm{ZnFe}_{2} \mathrm{O}_{4}$ spinel ferrite nanoparticles with different inversion degrees: a comparative study, Inorg. Chem. Front., 2019, 6, 1527-1534.

29 J. Ding, P. G. McCormick and R. Street, Magnetic properties of mechanically alloyed $\mathrm{CoFe}_{2} \mathrm{O}_{4}$, Solid State Commun., 1995, 95, 31-33.

30 Y. Shi, J. Ding and H. Yin, $\mathrm{CoFe}_{2} \mathrm{O}_{4}$ nanoparticles prepared by the mechanochemical method, J. Alloys Compd., 2000, 308, 290-295.

31 C. N. R. Rao, A. Müller and A. K. Cheetham, The chemistry of nanomaterials Synthesis, properties and applications, WileyVCH, Weinheim, 2004.

32 J. S. Jiang, L. Gao, X. L. Yang, J. K. Guo and H. L. Shen, Nanocrystalline NiZn ferrite synthesized by high energy ball milling, J. Mater. Sci. Lett., 1999, 18, 1781-1783.

33 R. Sani, A. Beitollahi, Y. V Maksimov and I. P. Suzdalev, Synthesis, phase formation study and magnetic properties of $\mathrm{CoFe}_{2} \mathrm{O}_{4}$ nanopowder prepared by mechanical milling, $J$. Mater. Sci., 2007, 42, 2126-2131.

34 S. Komarneni, M. C. D'Arrigo, C. Leonelli, G. C. Pellacani and H. Katsuki, Microwave-Hydrothermal Synthesis of Nanophase Ferrites, J. Am. Ceram. Soc., 1998, 81, 3041-3043.

35 J. Zhou, J. Ma, C. Sun, L. Xie, Z. Zhao, H. Tian, Y. Wang, J. Tao and X. Zhu, Low-Temperature Synthesis of $\mathrm{NiFe}_{2} \mathrm{O}_{4}$ by a Hydrothermal Method, J. Am. Ceram. Soc., 2005, 88, 3535-3537.

36 P. Dolcet, S. Diodati, F. Zorzi, P. Voepel, C. Seitz, B. M. Smarsly, S. Mascotto, F. Nestola and S. Gross, Very fast crystallisation of $\mathrm{MFe}_{2} \mathrm{O}_{4}$ spinel ferrites $(\mathrm{M}=\mathrm{Co}, \mathrm{Mn}$, $\mathrm{Ni}, \mathrm{Zn}$ ) under low temperature hydrothermal conditions: a time-resolved structural investigation, Green Chem., 2018, 20, 2257-2268.

37 S. Diodati, L. Pandolfo, A. Caneschi, S. Gialanella and S. Gross, Green and low temperature synthesis of nanocrystalline transition metal ferrites by simple wet chemistry routes, Nano Res., 2014, 7, 1027-1042.

38 P. Anastas and N. Eghbali, Green Chemistry: Principles and Practice, Chem. Soc. Rev., 2010, 39, 301-312.

39 T. A. S. Ferreira, J. C. Waerenborgh, M. H. R. M. Mendonça, M. R. Nunes and F. M. Costa, Structural and morphological characterization of $\mathrm{FeCo}_{2} \mathrm{O}_{4}$ and $\mathrm{CoFe}_{2} \mathrm{O}_{4}$ spinels prepared by a coprecipitation method, Solid State Sci., 2003, 5, 383392.

40 P. Debye and P. Scherrer, Atomaufbau, 1918, 101-120.

41 L. B. Mccusker, R. B. Von Dreele, D. E. Cox, D. Loue and P. Scardi, Rietveld refinement guidelines, J. Appl. Crystallogr., 1999, 32, 36-50.

42 R. A. Young, The Rietveld method, International Union of Crystallography, Oxford University Press, Chester, England, Oxford, New York, 1993.

43 J. T. S. Irvine, D. C. Sinclair and A. R. West, Electroceramics: Characterization by Impedance Spectroscopy, Adv. Mater., 1990, 2, 132-138.

44 C. Murugesan, M. Perumal and G. Chandrasekaran, Structural, dielectric and magnetic properties of cobalt ferrite prepared using auto combustion and ceramic route, Phys. B, 2014, 448, 53-56.

45 C. Murugesan, L. Okrasa and G. Chandrasekaran, Structural, AC conductivity, impedance and dielectric study of nanocrystalline $\mathrm{MFe}_{2} \mathrm{O}_{4}(\mathrm{M}=\mathrm{Mg}$, Co or $\mathrm{Cu})$ spinel ferrites, J. Mater. Sci.: Mater. Electron., 2017, 28, 13168-13175.

$46 \mathrm{H}$. L. Tuller, Ionic conduction in nanocrystalline materials, Solid State Ionics, 2000, 131, 143-157.

47 J. Maier, On the Conductivity of Polycrystalline Materials, Berichte der Bunsengesellschaft für physikalische Chemie, 1986, 90, 26-33.

48 J. J. Neumeier, M. T. Elm, B. Luerßen and J. Janek, Platinum microelectrodes on gadolinia doped ceria single crystals bulk properties and electrode kinetics, Phys. Chem. Chem. Phys., 2018, 20, 8294-8301.

49 M. T. Elm, J. D. Hofmann, C. Suchomski, J. Janek and T. Brezesinski, Ionic Conductivity of Mesostructured YttriaStabilized Zirconia Thin Films with Cubic Pore Symmetry On the Influence of Water on the Surface Oxygen Ion Transport, ACS Appl. Mater. Interfaces, 2015, 7, 11792-11801.

$50 \mathrm{X}$. Guo and Z. Zhang, Grain size dependent grain boundary defect structure: case of doped zirconia, Acta Mater., 2003, 51, 2539-2547.

$51 \mathrm{~J}$. Fleig, The grain boundary impedance of random microstructures: numerical simulations and implications for the analysis of experimental data, Solid State Ionics, 2002, 150, 181-193.

52 A. Tschöpe, E. Sommer and R. Birringer, Grain sizedependent electrical conductivity of polycrystalline cerium oxide: I. Experiments, Solid State Ionics, 2001, 139, 255-265.

53 A. Tschöpe, J. Y. Ying and H. L. Tuller, Catalytic redox activity and electrical conductivity of nanocrystalline nonstoichiometric cerium oxide, Sens. Actuators, B, 1996, 31, 111-114.

54 A. Tschöpe and R. Birringer, Grain Size Dependence of Electrical Conductivity in Polycrystalline Cerium Oxide, $J$. Electroceram., 2001, 7, 169-177.

55 W. Kündig and R. Steven Hargrove, Electron hopping in magnetite, Solid State Commun., 1969, 7, 223-227.

56 F. Walz, The Verwey transition - a topical review, J. Phys.: Condens. Matter, 2002, 14, R285-R340. 
57 G. H. Jonker, Analysis of the semiconducting properties of cobalt ferrite, J. Phys. Chem. Solids, 1959, 9, 165-175.

58 B. Gillot and F. Jemmali, Dependence of electrical properties in iron-cobalt, iron-zinc ferrites near stoichiometry on firing temperature and atmosphere, Phys. Status Solidi, 1983, 76, 601-608.

59 J. Smit and H. P. J. Wijn, Ferrites, Philips Technical Library, 1959.

60 D. Venkateshvaran, M. Althammer, A. Nielsen, S. Geprägs, M. S. Ramachandra Rao, S. T. B. Goennenwein, M. Opel and R. Gross, Epitaxial $\mathrm{Zn}_{x} \mathrm{Fe}_{3-x} \mathrm{O}_{4}$ thin films: a spintronic material with tunable electrical and magnetic properties, Phys. Rev. B: Condens. Matter Mater. Phys., 2009, 79, 134405.

61 D. Tripathy, A. O. Adeyeye, C. B. Boothroyd and S. N. Piramanayagam, Magnetic and transport properties of Co-doped $\mathrm{Fe}_{3} \mathrm{O}_{4}$ films, J. Appl. Phys., 2007, 101, 13904.

62 L. Ajroudi, N. Mliki, L. Bessais, V. Madigou, S. Villain and C. Leroux, Magnetic, electric and thermal properties of cobalt ferrite nanoparticles, Mater. Res. Bull., 2014, 59, 4958.

63 S. Kumar, S. Munjal and N. Khare, Metal-semiconductor transition and Seebeck inversion in $\mathrm{CoFe}_{2} \mathrm{O}_{4}$ nanoparticles, J. Phys. Chem. Solids, 2017, 105, 86-89. 